\title{
Fractal Behavior in the Clarification Process of Cane Sugar Production
}

\author{
Xiaomo Yu, ${ }^{1}$ Junke Ye, ${ }^{2}$ Jing Hu, ${ }^{3,4}$ Xiaoping Liao, ${ }^{2}$ and Jianbo Gao ${ }^{3,4}$ \\ ${ }^{1}$ College of Light Industry and Food Engineering, Guangxi University, 100 Daxue Road, Nanning, Guangxi 530005, China \\ ${ }^{2}$ College of Mechanical Engineering, Guangxi University, 100 Daxue Road, Nanning, Guangxi 530005, China \\ ${ }^{3}$ Institute of Complexity Science and Big Data Technology, Guangxi University, 100 Daxue Road, Nanning, Guangxi 530005, China \\ ${ }^{4}$ PMB Intelligence LLC, Sunnyvale, CA 94087, USA
}

Correspondence should be addressed to Jianbo Gao; jbgao.pmb@gmail.com

Received 2 September 2013; Accepted 6 October 2013

Academic Editor: Ahmed El Wakil

Copyright (C) 2013 Xiaomo Yu et al. This is an open access article distributed under the Creative Commons Attribution License, which permits unrestricted use, distribution, and reproduction in any medium, provided the original work is properly cited.

\begin{abstract}
Cane sugar production is an important industrial process. One of the most important steps in cane sugar production is the clarification process, which provides high-quality, concentrated sugar syrup crystal for further processing. To gain fundamental understanding of the physical and chemical processes associated with the clarification process and help design better approaches to improve the clarification of the mixed juice, we explore the fractal behavior of the variables pertinent to the clarification process. We show that the major variables in this key process all show persistent long-range correlations, for time scales up to at least a few days. Persistent long-range correlations amount to unilateral deviations from a preset target. This means that when the process is in a desired mode such that the target variables, color of the produced sugar and its clarity degree, both satisfy preset conditions, they will remain so for a long period of time. However, adversity could happen, in the sense that when they do not satisfy the requirements, the adverse situation may last quite long. These findings have to be explicitly accounted for when designing active controlling strategies to improve the quality of the produced sugar.
\end{abstract}

\section{Introduction}

For centuries, sugar has been a highly regarded and widely traded commodity, for the simple reason that sweetness is an essential ingredient of life. Before 1990, sugar beet accounted for about $40 \%$ of the total sugar production in the world. Since then, cane sugar market has been rapidly expanding, due to the low cost in sugarcane production. According to the US Department of Agriculture, the global cane sugar production reached 117.5 million tons in 2009, amounting to $78.33 \%$ of the total sugar production. Among the major cane sugar producer and consumer, China ranks the 3rd, accounting for $7 \%$ of the world totalproduction.

In general, cane sugar manufacturing consists of six phases: milling, clarification, evaporation, crystallization, centrifuging, and drying so as to obtain the white sugar, brown sugar, and other products. In particular, the juice from the milling workshop is called mixed juice.
Besides water, sugarcane, and reduced sugar, the mixed juice also contains many organic and inorganic nonsugar components, such as colloidal substances, inorganic salts (iron, magnesium, aluminum, calcium, etc.), and pigments. While these nonsugar components are residual nutrients in the sugarcane, they are detrimental to the sugar production. For example, flavonoids, multiacid, and organic-acid can make the mixed juice appear dark brown. Since the nonsugar components affect not only the appearance and color, but also the concentration of the sugar (i.e., reflected as the sweetness of sugar by a consumer), they have to be carefully removed. The purpose of the clarification process is to remove as many nonsugar components as possible, improve the purity of the juice, and reduce its viscosity and color values. This is critical for providing high-quality, concentrated sugar syrup crystal to the boiling stage. Therefore, the clarification stage is a key process in cane sugar production. 
There have been efforts to improve the clarification of the mixed juice by using neural network-based prediction schemes $[1,2]$. Such approaches amount to treating the clarification process as a black or gray box and thus do not yield any understanding of the basic physics and chemistry involved in clarification. To help gain more fundamental understanding and guide the design of better approaches to improve clarification, in this paper, we carry out fractal analysis of the key variables pertinent to the clarification process.

Among the types of activity that characterize complex systems, the most ubiquitous and puzzling is perhaps the appearance of $1 / f^{\alpha}$ noise, a form of temporal or spatial fluctuation characterized by a power-law decaying power spectral density. A subclass of such processes, denoted as $1 / f^{2 H+1}$, is called processes with long-range correlations (or long memories) characterized by a Hurst parameter $H$. Depending on whether $0<H<1 / 2, H=1 / 2$, or $1 / 2<H<1$ [3], they are said to have antipersistent correlations, memoryless or only short-range correlations, or persistent long-range correlations. Prominent examples of such processes include vision [4], finance [5], DNA sequences [6-10], human cognition [11] and coordination [12], posture [13], cardiac dynamics [14-17], and the distribution of prime numbers [18], to name but a few. In this paper, we wish to explore whether fractal theory can shed new light on the clarification process of cane sugar production.

The remainder of the paper is organized as follows. In Section 2, we provide some details about the cane sugar clarification process, explain the various variables to be analyzed here, and discuss the challenges of analyzing those variables. In Section 3, we carry out fractal analysis of these variables using the key concept in random fractal theory, the Hurst parameter, which characterizes the basic correlation structure of the signals. Concluding discussions are presented in Section 4.

\section{Clarification of Mixed Juice in Cane Sugar Production}

Sugar clarification is a key process in cane sugar production. It is characterized by two important indices called color value and clarity degree. Whether the color value and the clarity degree can achieve the desired or preset values can critically affect the quality of the cane sugar and the revenue of a factory.

Many methods have been developed for clarifying the mixed juice. The two major ones are the carbonation method and the sulphitation method. The former uses the milk of lime and carbon dioxide as main detergent on the mixed juice. Besides high cost, the accompanying pollution by carbonated mud is hard to deal with. Therefore, its usage has been substantially reduced over the years.

Unlike the carbonate method, the sulphitation method adds clarifying agent $\mathrm{SO}_{2}$ in addition to lime and phosphoric acid. Due to the absorption effect of calcium sulfite (which is the product of lime and $\mathrm{SO}_{2}$ ), together with the inhibition of pigment formation due to $\mathrm{SO}_{2}$, the sulphitation method is significantly better than the carbonate method. In the following we will focus on the sulphitation method for the clarification of the mixed juice.

The sulphitation method for clarification is schematically shown in Figure 1. It is a complicated physical chemical process, involving solvents such as $\mathrm{SO}_{2}$, milk of lime and phosphoric acid. Roughly, the process can be divided into four stages, cane juice phosphorus-preliming, first heating, $\mathrm{SO}_{2}$ sulphitation-neutralization and settlement after 2ndheating. More specifically, in the preliming stage after the first heating to $55 \sim 65^{\circ} \mathrm{C}$, milk of lime and phosphoric acid are added to the mixed sugar cane juice. In the next sulphur fumigation process, $\mathrm{SO}_{2}$ gas enters the chemical process pipeline. At the end of the pipeline, milk of lime is further added for achieving neutralization, since phosphoric acid sulfite reacts with calcium hydroxide to form calcium phosphate and calcium sulfate. Then, the 2 nd heating is carried out so that sugar cane colloids of mixed juice can not only fully condense in the precipitator, but also (1) accelerate precipitation, (2) decrease juice viscosity, and (3) facilitate the precipitated particles to sink fast. Then, the clarified juice can come out of the settler from the top of each later, while the mud juice is discharged into the vacuum suction filter by gravity. Filtered juice can then be directly incorporated into the clear juice heating device and sent to the evaporation device to condense into syrup. Therefore, controlling the process parameters steadily is the key to improve the quality of clarified juice and sugar.

In this paper, time series of the 11 variables indicated in Figure 1 will be analyzed using random fractal theory. The data were measured in an advanced cane sugar production factory in Guangxi Zhuang Autonomous Region of China. Guangxi is the largest sugar producer in China, amounting to more than $60 \%$ of the total sugar in the country. The data were collected with a time interval of 2 hours and lasted for the whole season (about 4 months). These variables are grouped into three groups, with the last two variables, $x_{10}$, color value, and $x_{11}$, the clarity degree, being the target variables. The time series data of these 11 variables are shown in Figures 2, 3, and 4. As can be seen, the major challenge in analyzing these data is nonstationarity; that is, the statistical moments, including mean and variance, vary with time. Understandably, this may be a salient feature for variables that are measured over a very long period of time (which is about 4 months here).

\section{Fractal Analysis of the Clarification Process}

In this section, we first overview the basics of fractal processes with long memory and then briefly describe two methods for detecting fractal structures from data with trends. One is the seminal detrended fluctuation analysis (DFA) [19]. The other is adaptive fractal analysis (AFA) [20-23]. Since AFA provides additional advantages over DFA to deal with signals with arbitrary trends, we only present results of AFA for the variables pertinent to the clarification process of cane sugar production here.

3.1. Basics of Fractal Processes with Long Memory. As we have mentioned, fractal processes with long memory are characterized by the Hurst parameter $H$. To better understand the 


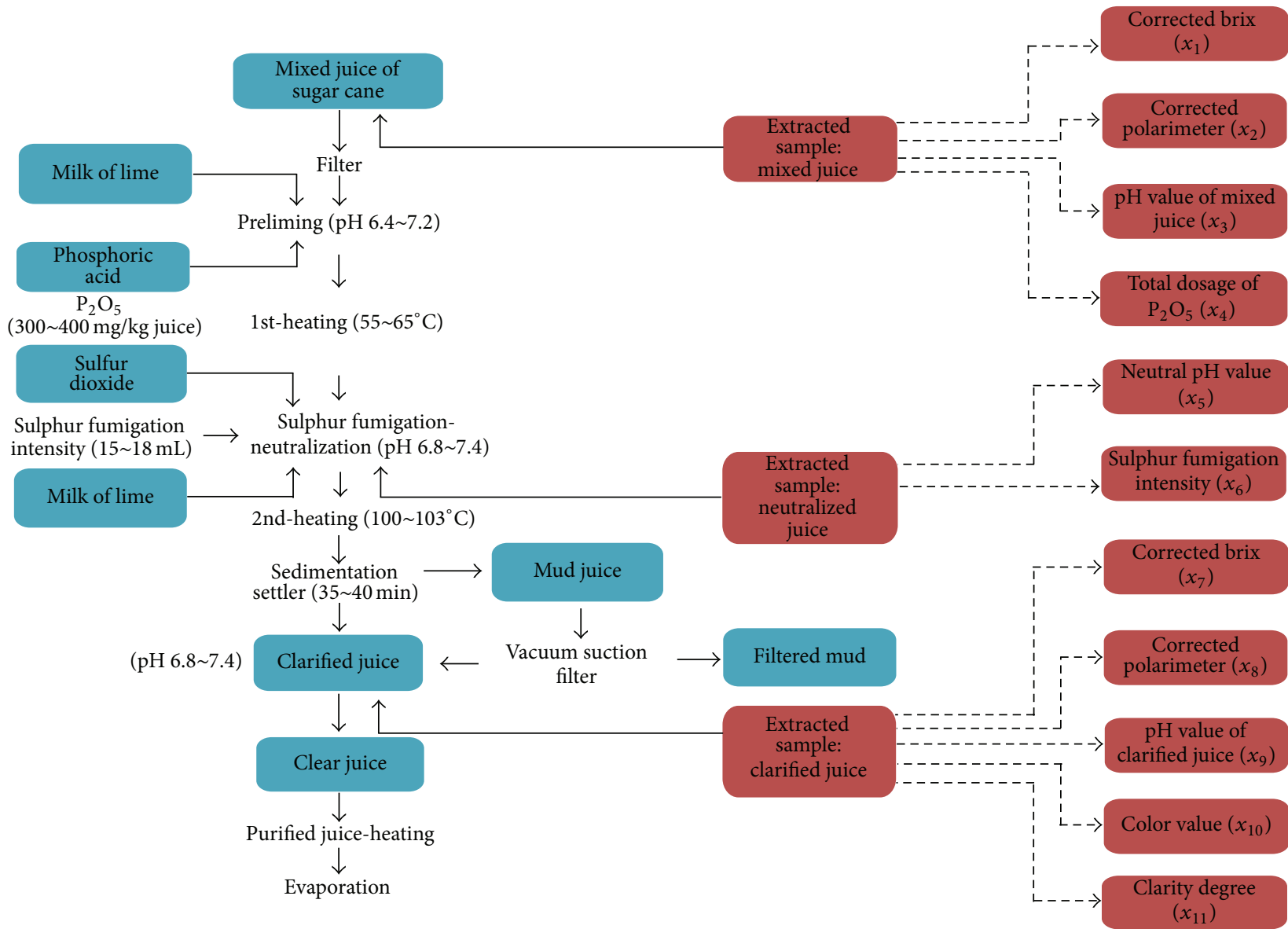

FIGURE 1: Schematic of the clarification stage of cane sugar production. Variables $x_{i}, i=1,2, \ldots, 11$, are analyzed here.

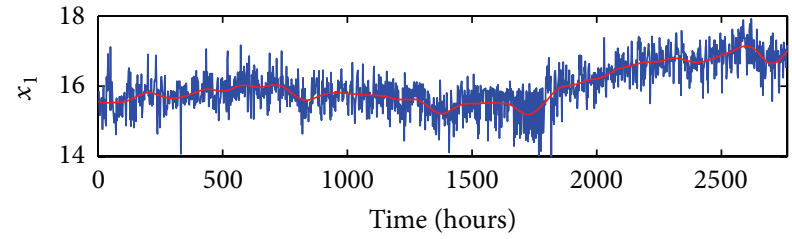

(a)

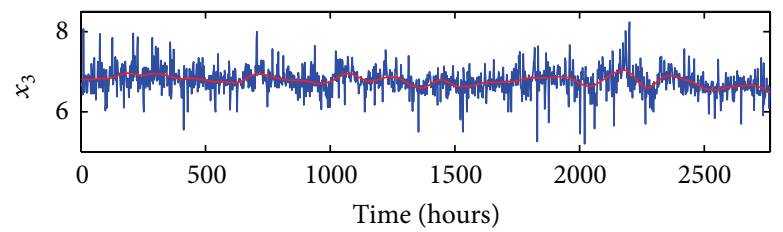

(c)

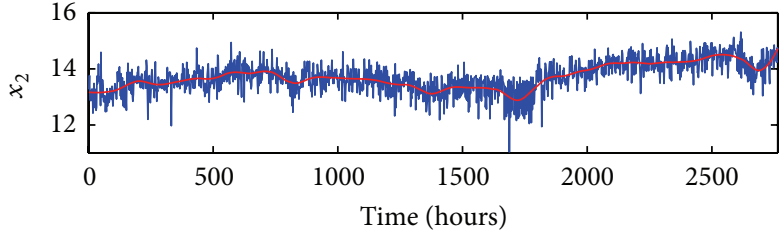

(b)

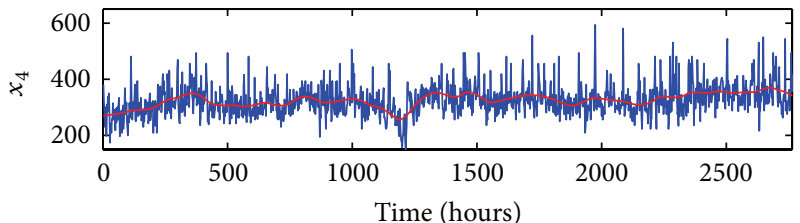

(d)

FIGURE 2: Signals $x_{1}$ to $x_{4}$.

meaning of $H$, it is useful to mathematically be more precise. Let $\left\{x_{1}, x_{2}, \ldots, x_{n}\right\}$ be a stationary stochastic process with mean $\bar{x}$ and autocorrelation function of the type

$$
r(k) \sim k^{2 H-2}, \quad \text { as } k \longrightarrow \infty,
$$

where $0<H<1$ is the Hurst parameter. When $1 / 2<H<$ $1, \sum_{k} r(k)=\infty$, leading to the term long range correlation. $\left\{x_{1}, x_{2}, \ldots, x_{n}\right\}$ is often called an increment (or noise) process. Its power spectral density (PSD) is $1 / f^{2 H-1}$. Its integration,

$$
u(i)=\sum_{k=1}^{i}\left(x_{k}-\bar{x}\right), \quad i=1,2, \ldots, n,
$$

is called a random walk process having PSD $1 / f^{2 H+1}$. Being $1 / f$ processes, they cannot be aptly modeled by Markov 


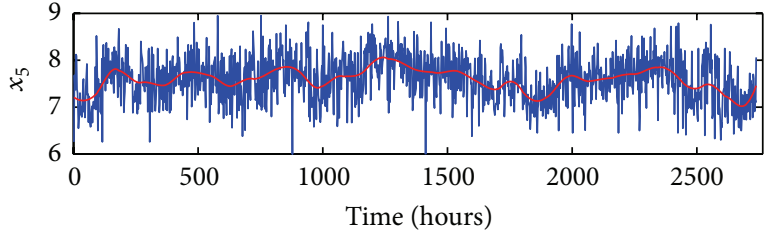

(a)

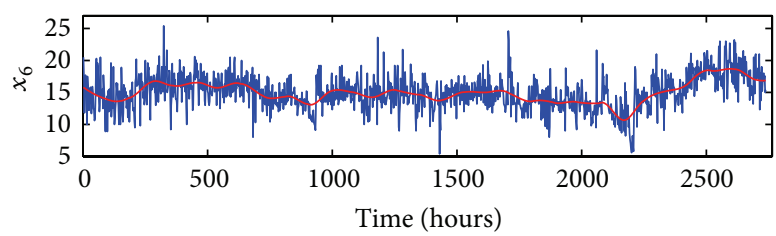

(b)

FIGURE 3: Signals $x_{5}$ and $x_{6}$.

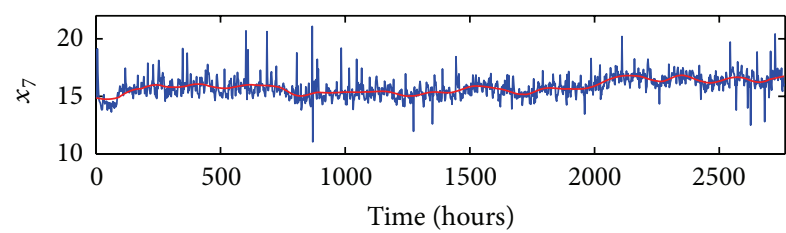

(a)

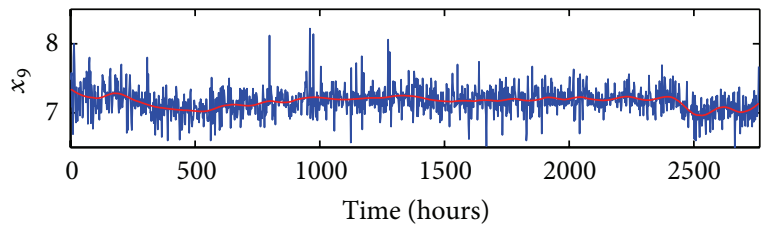

(c)

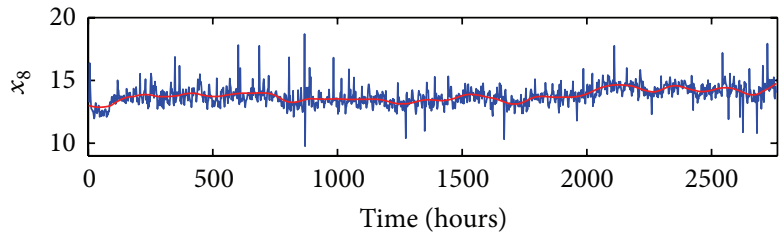

(b)

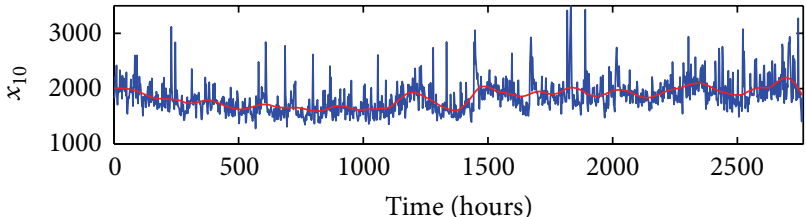

(d)

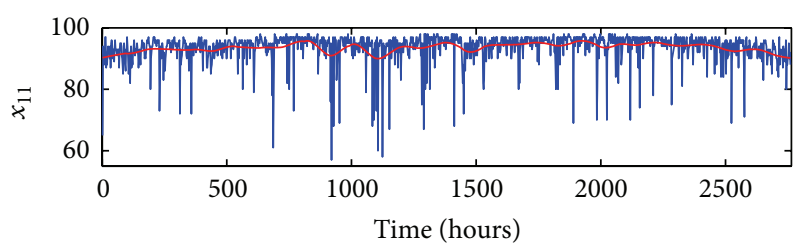

(e)

Figure 4: Signals $x_{7}$ to $x_{11}$.

processes or ARIMA models [24], since the PSD for those processes is distinctly different from $1 / f$. To adequately model $1 / f$ processes, fractional order processes have to be used. The most popular is the fractional Brownian motion model [3].

To deepen our understanding of the Hurst parameter, let us smooth $\left\{x_{1}, x_{2}, \ldots, x_{n}\right\}$ using nonoverlapping windows to yield a new time series

$$
X_{t}^{(m)}=\frac{\left(x_{t m-m+1}+\cdots+x_{t m}\right)}{m}, \quad t \geq 1 .
$$

It can be proven that the variance of the new time series is given by [25]

$$
\operatorname{var}\left(X^{(m)}\right)=\sigma^{2} m^{2 H-2},
$$

where $\sigma^{2}$ is the variance of original stochastic process $\left\{x_{1}, x_{2}, \ldots, x_{n}\right\}$. Equation (4) offers an excellent means of understanding $H$. For example, if $H=0.50, m=100$, then $\operatorname{var}\left(X^{(m)}\right)=\sigma^{2} / 100$. When $H=0.75$, in order to have $\operatorname{var}\left(X^{(m)}\right)=\sigma^{2} / 100$, then we need $m=10^{4}$, which is much larger than $m=100$ for the case of $H=0.50$. On the other hand, when $H=0.25$, if we still want $\operatorname{var}\left(X^{(m)}\right)=\sigma^{2} / 100$, then $m \approx 21.5$, much smaller than $m=100$, the case of $H=0.50$. An interesting lesson from such a simple discussion is that if a time series is short while its $H$ is close to 1 , then smoothing is not a viable option for reducing the variations there.

There are many excellent methods for estimating $H[25$, 26]. In the next two subsections, we describe the methods that are most promising for detecting fractal variations in variables pertinent to the clarification process of cane sugar production.

3.2. Detrended Fluctuation Analysis (DFA). Denote a time series of interest by $x(1), x(2), x(3), \ldots$; DFA works as follows [19]. First divide a given time series of length $N$ into $\lfloor N / l\rfloor$ nonoverlapping segments, each containing $l$ points. Then, define the local trend in each segment to be the ordinate of a linear least-squares fit or best polynomial fit of the time series in that segment; this is schematically shown in Figure 5 using a linear trend as an example. Finally, compute the "detrended 


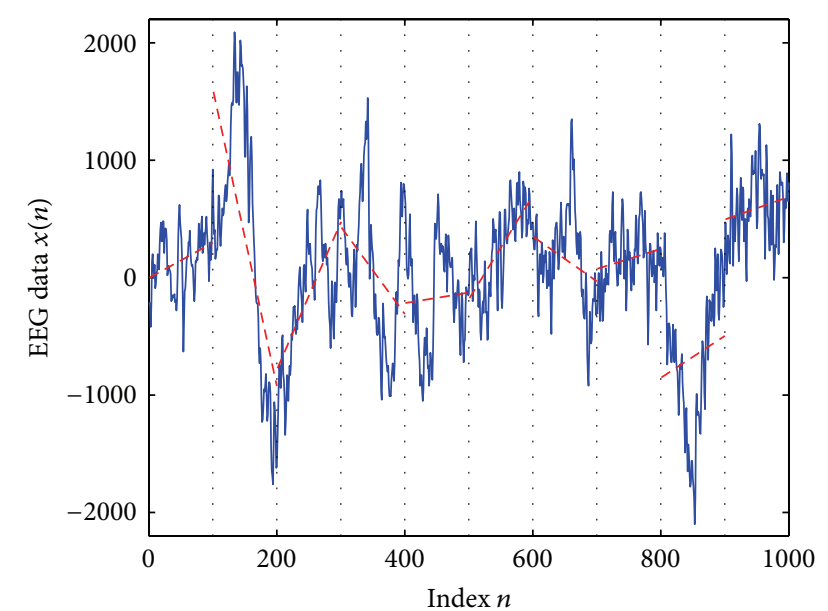

Figure 5: A schematic showing local detrending in the DFA method.

walk," denoted by $x_{l}(n)$, as the difference between the original "walk" $x(n)$ and the local trend. One then examines the following scaling behavior:

$$
F_{d}(l)=\left\langle\sum_{i=1}^{l} x_{l}(i)^{2}\right\rangle \sim l^{2 H},
$$

where the angle brackets denote ensemble averages of all the segments.

Note that the best linear or polynomial fits of DFA may have large discontinuities at the boundaries of adjacent segments. This is clearly shown in Figure 5. Such discontinuities prevent DFA from effectively removing a complicated trend such as the 11-year cycle in sunspot numbers [27]. As a result, DFA alone may not be effective enough to detect fractal variations from data with trends.

3.3. Adaptive Fractal Analysis (AFA). AFA is built upon the adaptive detrending algorithm $[28,29]$. It fixes the potential problem of DFA by finding a globally smooth trend signal. Because of this, AFA has additional advantages over DFA $[20,21]$. For example, AFA can deal with arbitrary, strong nonlinear trends while DFA cannot [23, 27], AFA has better resolution of fractal scaling behavior for short time series [22], AFA has a direct interpretation in terms of spectral energy while DFA does not [23], and there is a simple proof of why AFA yields the correct $H$ while such a proof is not available for DFA.

To find the global trend, the method first partitions a time series into segments (or windows) of length $w=2 n+1$ points, where neighboring segments overlap by $n+1$ points, and thus introducing a time scale of $((w+1) / 2) \tau=(n+1) \tau$, where $\tau$ is the sampling time. For each segment, we fit a best polynomial of order $M$. Note that $M=0$ and 1 correspond to piecewise constant and linear fitting, respectively. Denote the fitted polynomial for the $i$ th and $(i+1)$ th segments by $y^{(i)}\left(l_{1}\right), y^{(i+1)}\left(l_{2}\right), l_{1}, l_{2}=1, \ldots, 2 n+1$, respectively. Note that the length of the last segment may be smaller than $2 n+1$. We define the fitting for the overlapped region as

$$
y^{(c)}(l)=w_{1} y^{(i)}(l+n)+w_{2} y^{(i+1)}(l), \quad l=1,2, \ldots, n+1,
$$

where $w_{1}=(1-(l-1) / n), w_{2}=(l-1) / n$ can be written as $\left(1-d_{j}\right) / n, j=1,2$, where $d_{j}$ denotes the distances between the point and the centers of $y^{(i)}$ and $y^{(i+1)}$, respectively. This means that the weights decrease linearly with the distance between the point and the center of the segment. Such a weighting ensures symmetry and effectively eliminates any jumps or discontinuities around the boundaries of neighboring segments. In fact, the scheme ensures that the fitting is continuous everywhere, is smooth at the nonboundary points, and has the right- and left-derivatives at the boundary. The method can effectively determine any kind of trend signal. Those for the 11 variables studies here are shown as red curves in Figures 2-4.

Now we can describe AFA. If we start from an increment process, $x(1), x(2), \ldots$. Similar to DFA, we first construct a random walk process using (2). If the original data can be considered as a random walk-like process, which is true for EEG $[25,30,31]$, and sea clutter radar returns $[26,32,33]$, then this step is not necessary. However, for ideal fractal processes, there is no penalty if this is done, even though the process is already a random walk process.

Next, for a window size $w$, we determine, for the random walk process $u(i)$ (or the original process if it is already a random walk process), a global trend $v(i), i=1,2, \ldots, N$. Here, $N$ is the length of the random walk process. The residual, $u(i)-v(i)$, characterizes fluctuations around the global trend, and its variance yields the Hurst parameter $H$ [23]:

$$
F^{(2)}(w)=\left[\frac{1}{N} \sum_{i=1}^{N}(u(i)-v(i))^{2}\right]^{1 / 2} \sim w^{H} .
$$

3.4. Fractal Variations in the Clarification Process of Cane Sugar Production. Let us first focus on the group 1 variables, $x_{i}, i=1,2,3,4$, which represent corrected brix, corrected polarimeter, $\mathrm{pH}$ value of the mixed juice, and the total dosage of $\mathrm{P}_{2} \mathrm{O}_{5}$, respectively. Their AFA curves are shown in Figures $6(a)-6(d)$. We observe that there are two scaling regions for $x_{1}$ : on time scales shorter than $2^{5} \times 2=64$ hours, the Hurst parameter is 0.74 . On time scales greater than 64 hours, the Hurst parameter becomes 1.42. This means that, for time scales smaller than about 3 days, the variation in $x_{1}$ has persistent long-range correlations. On time scales longer than about 3 days, the signal becomes very non-stationary$H>1$ is often associated with non-stationarity $[25,26]$. The behavior of $x_{2}$ is very similar to that of $x_{1}$. Indeed, one can clearly observe that the signal of $x_{2}$ looks very similar to that of $x_{1}$. However, the behaviors of $x_{3}$ and $x_{4}$ are quite different from those of $x_{1}$ and $x_{2}$. In fact, they have better fractal scaling behavior (i.e., straighter linear relations in $\log _{2} F(w)$ versus $\log _{2} w$ plots) and stronger persistent correlations.

Next, we consider the variables $x_{5}$ and $x_{6}$, the neutral $\mathrm{pH}$ value, and the sulphur fumigation intensity. Their AFA curves 


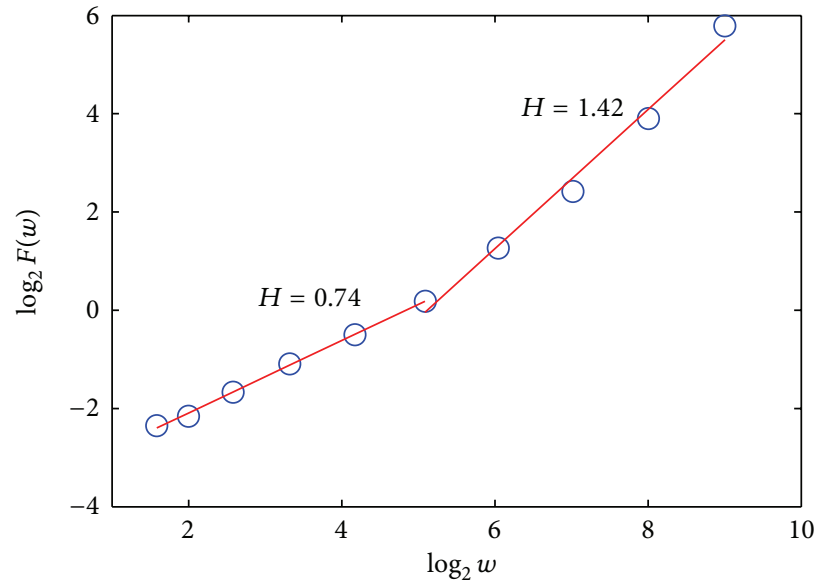

(a)

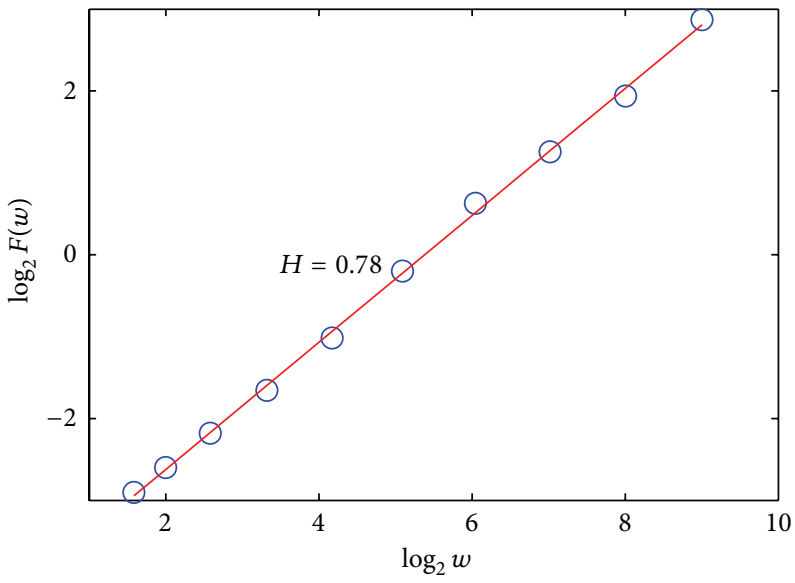

(c)

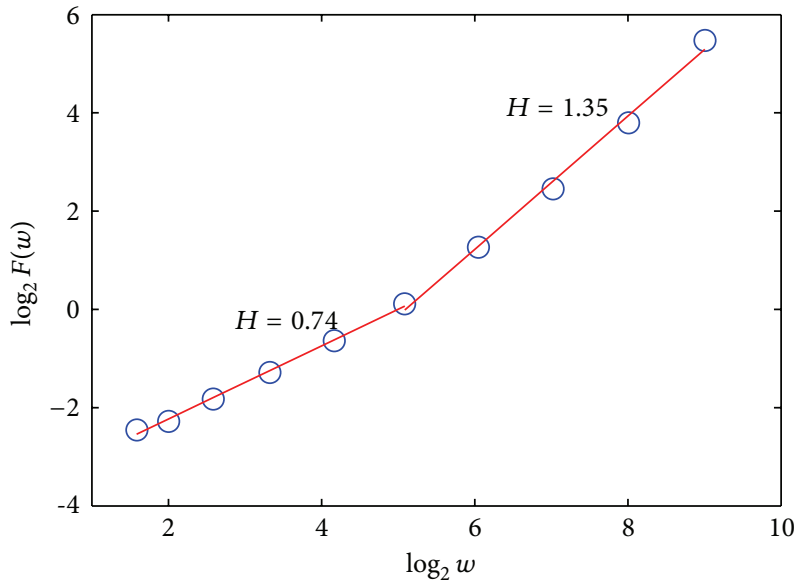

(b)

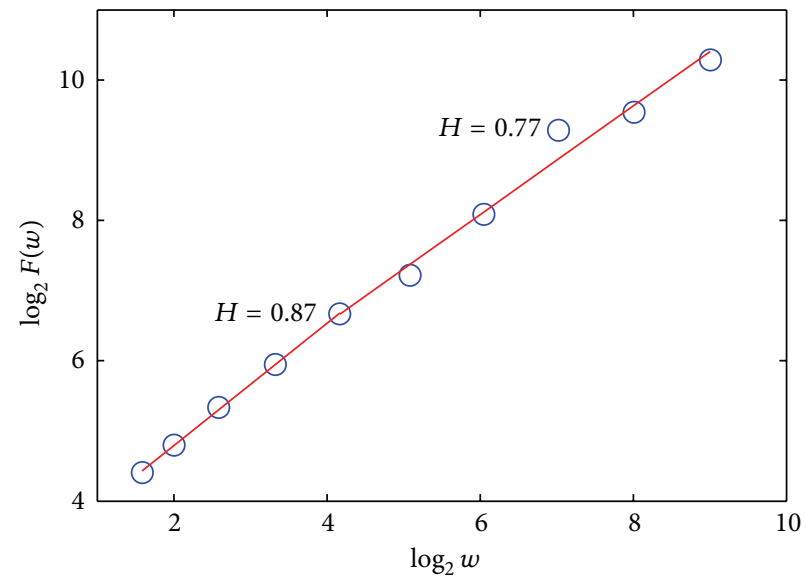

(d)

Figure 6: Adaptive fractal analysis (AFA) of signals $x_{1}$ to $x_{4}: \log _{2} F(w)$ versus $\log _{2} w$ for (a) $x_{1}$, (b) $x_{2}$, (c) $x_{3}$, and (d) $x_{4}$.

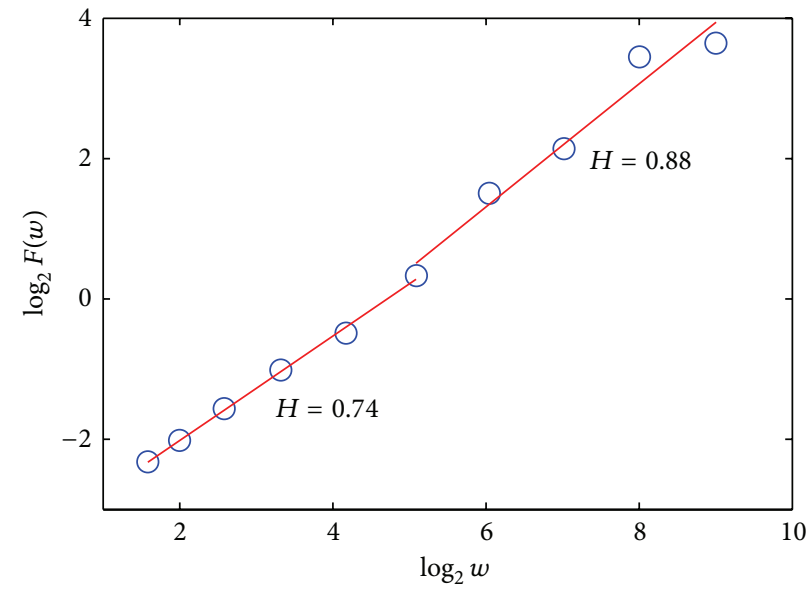

(a)

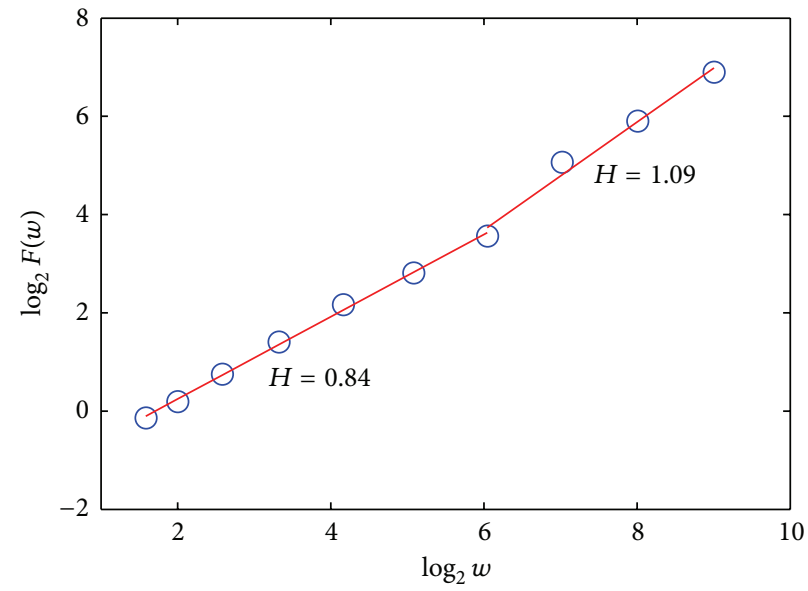

(b)

FIGURE 7: Adaptive fractal analysis (AFA) of signals (a) $x_{5}$ and (b) $x_{6}$. 


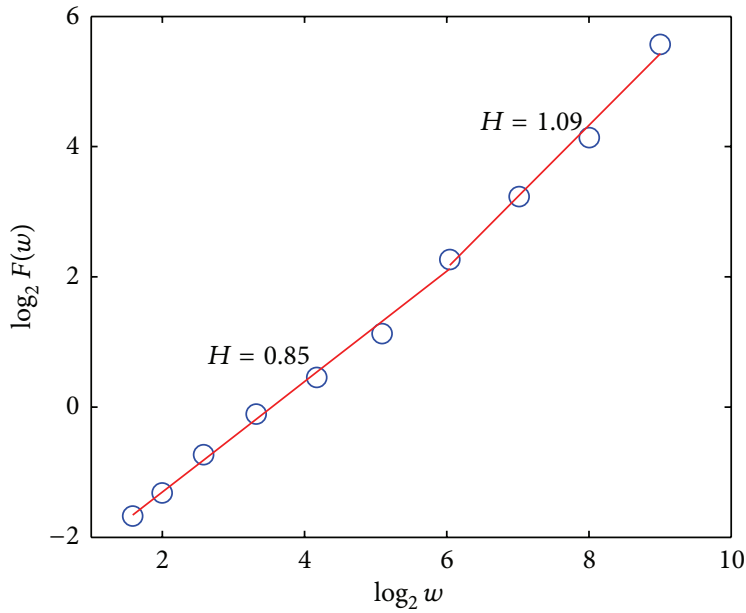

(a)

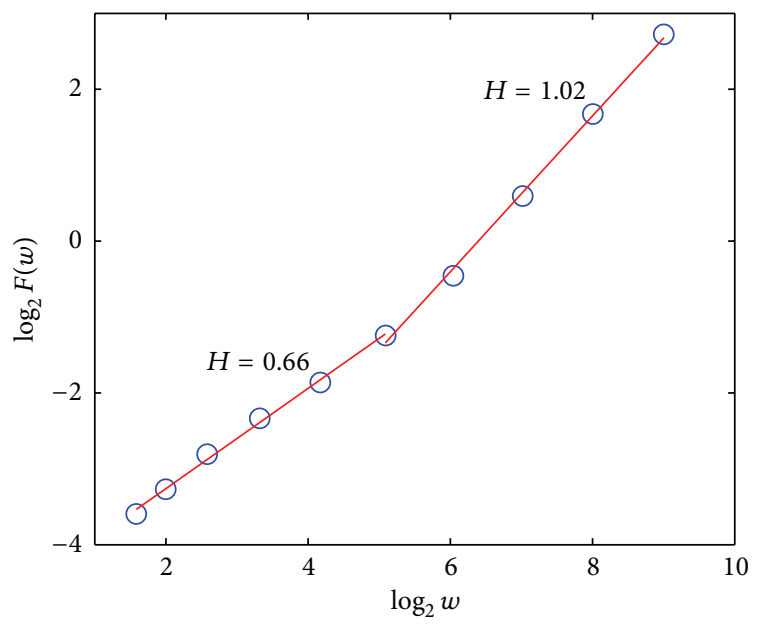

(c)

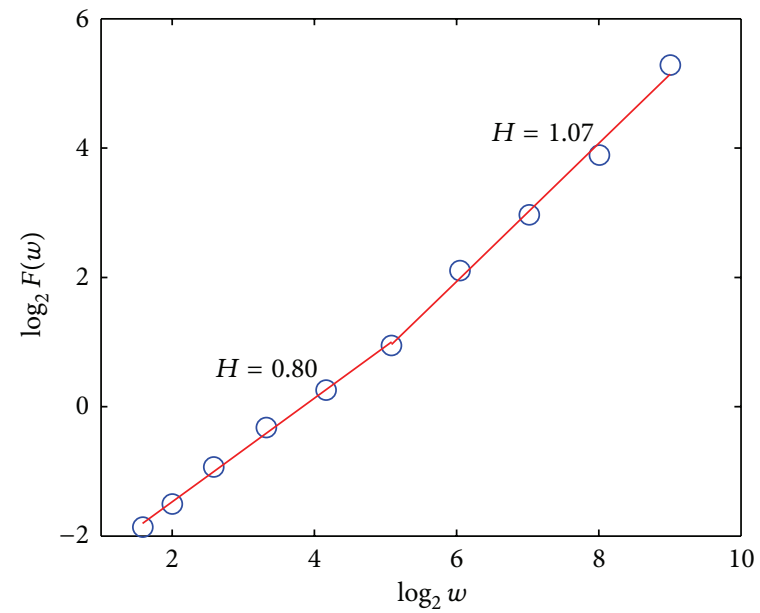

(b)

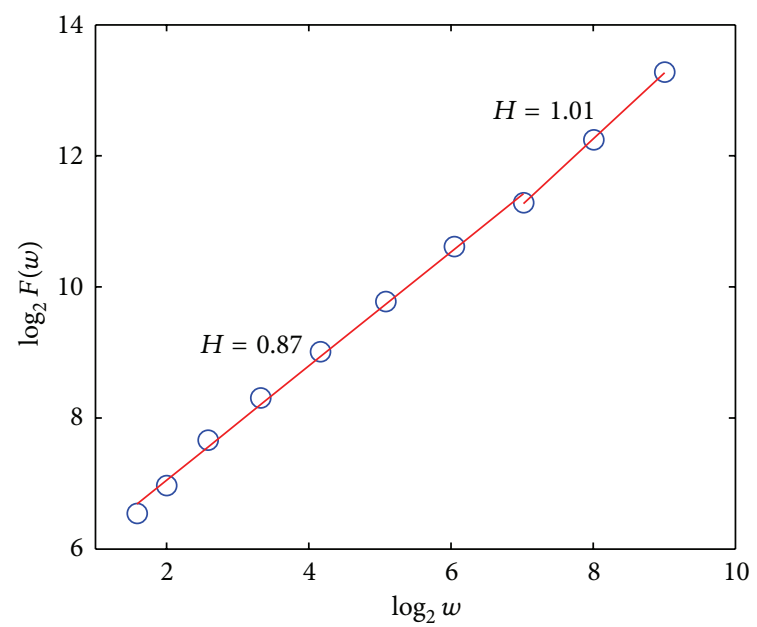

(d)

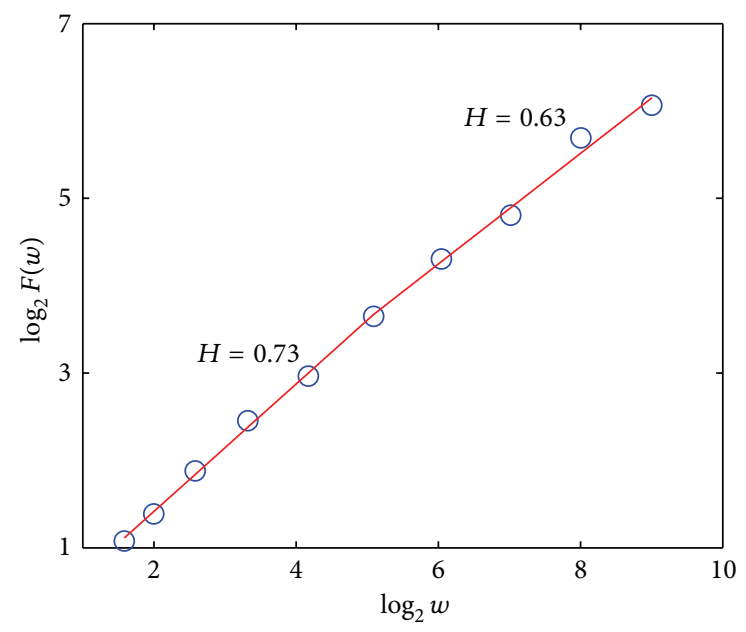

(e)

FIgURE 8: Adaptive fractal analysis (AFA) of signals (a) $x_{7}$, (b) $x_{8}$, (c) $x_{9}$, (d) $x_{10}$, and (e) $x_{11}$. 
are shown in Figure 7. They also have two scaling regions, and persistent correlations on short time scales (about 64 hours for $x_{5}$ and 128 hours for $x_{6}$ ). On longer time scales, the correlation in $x_{5}$ becomes more persistent ( $H$ changes from 0.74 to 0.88 ), while $x_{6}$ becomes non-stationary, since $H$ becomes larger than 1 .

Third, we consider $x_{i}, i=7,8, \ldots, 11$, which represents the corrected brix, corrected polarimeter, the $\mathrm{pH}$ value for the clarified juice after the 2nd heating, the color value, and the clarity degree, respectively. Their AFA curves are shown in Figure 8. We first examine $x_{i}, i=7,8$, 9. It is instructive to compare the curves in Figures $8(\mathrm{a})-8(\mathrm{c})$ with those in Figures 6(a)-6(c). In particular, we observe that, for the brix and polarimeter, on shorter time scales, the correlations become more consistent, since now $H$ becomes bigger. However, on longer time scales, $H$ becomes only slightly larger than 1 . Therefore, the degree of nonstationarity has decreased. The behavior of the $\mathrm{pH}$ value becomes more complicated than that in Figure 6, since there are two scaling regions in Figure 8(c), but there is only one scaling behavior in Figure 6(c).

Finally, we examine the AFA curves for the two most important variables, color value $x_{10}$, and clarity degree $x_{11}$, which are shown in Figures $8(\mathrm{~d})$ and $8(\mathrm{e})$. We observe that, for $x_{10}$, the first scaling, with $H=0.87$, is up to a time scale $2^{7} \times 2=256$ hours. Beyond that time scale, $H$ becomes 1 ; therefore, the signal becomes marginally nonstationary. The clarify degree, on the other hand, has an $H=0.73$ for time scales up to about 64 hours, and $H=0.63$ for longer time scales.

In summary, we have observed that persistent correlations up to a few days are salient features of the 11 variables that are most important for the clarification process of cane sugar production.

\section{Concluding Discussions}

Cane sugar production is an important industrial process. One of the most important steps in cane sugar production is the clarification process, which provides high-quality, concentrated sugar syrup crystal for further processing. To gain fundamental understanding of the physical and chemical processes associated with the clarification process and help design better approaches to improve the clarification of the mixed juice, in this paper, we have examined the fractal behavior of the 11 variables pertinent to the clarification process. We have shown that they all show persistent longrange correlations, for time scales up to at least a few days. Persistent long-range correlations amount to unilateral deviations from a preset target. This means that when the process is in a desired mode such that the target variables, color of the produced sugar and its clarity degree, both satisfy preset conditions, they will remain so for a long period of time. However, adversity could happen, in the sense that when they do not satisfy the requirements, the adverse situation may last quite long. These findings have to be explicitly accounted for when designing active controlling strategies to improve the quality of the produced sugar.

\section{Conflict of Interests}

The authors declare no conflict of interests.

\section{Acknowledgment}

This work (X. Yu and X. Liao) was partially supported by the Chinese Natural Science Foundation project number 50965003.

\section{References}

[1] S. Kamat, V. Diwanji, J. G. Smith, and K. P. Madhavan, "Modeling of $\mathrm{pH}$ process using recurrent neural network and wavenet," in Proceedings of the IEEE International Conference on Computational Intelligence for Measurement Systems and Applications (CIMSA '05), pp. 209-214, Giardini Naxos, Italy, July 2005.

[2] X. Lin, J. Yang, H. Liu, S. Song, and C. Song, "An improved method of DHP for optimal control in the clarifying process of sugar cane juice," in Proceedings of the International Joint Conference on Neural Networks (IJCNN '09), pp. 1814-1819, Atlanta, Ga, USA, June 2009.

[3] B. B. Mandelbrot, The Fractal Geometry of Nature, W. H. Freeman, San Francisco, Calif, USA, 1982.

[4] J. B. Gao, V. A. Billock, I. Merk et al., "Inertia and memory in ambiguous visual perception," Cognitive Processing, vol. 7, no. 2, pp. 105-112, 2006.

[5] J. Gao, J. Hu, W. Tung, and Y. Zheng, "Multiscale analysis of economic time series by scale-dependent lyapunov exponent," Quantitative Finance, vol. 13, no. 2, pp. 265-274, 2013.

[6] W. Li and K. Kaneko, "Long-range correlation and partial $1 / f^{\alpha}$ spectrum in a noncoding DNA sequence," Europhysics Letters, vol. 17, no. 7, pp. 655-660, 1992.

[7] R. F. Voss, "Evolution of long-range fractal correlations and $1 / f$ noise in DNA base sequences," Physical Review Letters, vol. 68, pp. 3805-3808, 1992.

[8] C.-K. Peng, S. V. Buldyrev, A. L. Goldberger et al., "Long-range correlations in nucleotide sequences," Nature, vol. 356, no. 6365, pp. 168-170, 1992.

[9] J. Gao, Y. Qi, Y. Cao, and W.-W. Tung, "Protein coding sequence identification by simultaneously characterizing the periodic and random features of DNA sequences," Journal of Biomedicine and Biotechnology, vol. 2005, no. 2, pp. 139-146, 2005.

[10] J. Hu, J.-B. Gao, Y. Cao, E. Bottinger, and W. Zhang, "Exploiting noise in array CGH data to improve detection of DNA copy number change," Nucleic Acids Research, vol. 35, no. 5, article e35, 2007.

[11] D. L. Gilden, T. Thornton, and M. W. Mallon, "1/f noise in human cognition," Science, vol. 267, no. 5205, pp. 1837-1839, 1995.

[12] Y. Chen, M. Ding, and J. A. Scott Kelso, "Long memory processes (1/ $f^{\alpha}$ type) in human coordination," Physical Review Letters, vol. 79, no. 22, pp. 4501-4504, 1997.

[13] J. J. Collins and C. J. de Luca, "Random walking during quiet standing," Physical Review Letters, vol. 73, no. 5, pp. 764-767, 1994.

[14] P. C. Ivanov, M. G. Rosenblum, C.-K. Peng et al., "Scaling behaviour of heartbeat intervals obtained by wavelet-based timeseries analysis," Nature, vol. 383, no. 6598, pp. 323-327, 1996.

[15] L. A. Nunes Amaral, A. L. Goldberger, P. C. Ivanov, and H. Eugene Stanley, "Scale-independent measures and pathologic 
cardiac dynamics," Physical Review Letters, vol. 81, no. 11, pp. 2388-2391, 1998.

[16] P. C. Ivanov, L. A. Nunes Amaral, A. L. Goldberger et al., "Multifractality in human heartbeat dynamics," Nature, vol. 399, no. 6735, pp. 461-465, 1999.

[17] P. Bernaola-Galván, F. C. Ivanov, L. A. Nunes Amaral, and H. E. Stanley, "Scale invariance in the nonstationarity of human heart rate," Physical Review Letters, vol. 87, no. 16, Article ID 168105, 4 pages, 2001.

[18] M. Wolf, " $1 / \mathrm{f}$ noise in the distribution of prime numbers," Physica A, vol. 241, no. 3-4, pp. 493-499, 1997.

[19] C.-K. Peng, S. V. Buldyrev, S. Havlin, M. Simons, H. E. Stanley, and A. L. Goldberger, "Mosaic organization of DNA nucleotides," Physical Review E, vol. 49, no. 2, pp. 1685-1689, 1994.

[20] M. A. Riley, N. Kuznetsov, S. Bonnette, S. Wallot, and J. B. Gao, "A tutorial introduction to adaptive fractal analysis," Frontiers in Fractal Physiology, 2012.

[21] N. Kuznetsov, S. Bonnette, J. B. Gao, and M. A. Riley, "Adaptive fractal analysis reveals limits to fractal scaling in center of pressure trajectories," Annals of Biomedical Engineering, vol. 41, no. 8, pp. 1646-1660, 2013.

[22] J. B. Gao, J. Hu, X. Mao, and M. Perc, "Culturomics meets random fractal theory: insights into long-range correlations of social and natural phenomena over the past two centuries," Journal of the Royal Society Interface, vol. 9, no. 73, pp. 19561964, 2012.

[23] J. Gao, J. Hu, and W.-W. Tung, "Facilitating joint chaos and fractal analysis of biosignals through nonlinear adaptive filtering," PLoS ONE, vol. 6, no. 9, Article ID e24331, 2011.

[24] G. E. P. Box and G. M. Jenkins, Time Series Analysis: Forecasting and Control, Holden-Day, San Francisco, Calif, USA, 1976.

[25] J. Gao, Y. Cao, W.-w. Tung, and J. Hu, Multiscale Analysis of Complex Time Series: Integration of Chaos and Random Fractal Theory, and BeyonD, Wiley-Interscience, New York, NY, USA, 2007.

[26] J. Gao, J. Hu, W.-W. Tung, Y. Cao, N. Sarshar, and V. P. Roychowdhury, "Assessment of long-range correlation in time series: how to avoid pitfalls," Physical Review E, vol. 73, no. 1, Article ID 016117, 2006.

[27] J. Hu, J. Gao, and X. Wang, "Multifractal analysis of sunspot time series: the effects of the 11-year cycle and fourier truncation," Journal of Statistical Mechanics, vol. 2009, no. 2, Article ID P02066, 2009.

[28] J. Gao, H. Sultan, J. Hu, and W.-W. Tung, "Denoising nonlinear time series by adaptive filtering and wavelet shrinkage: a comparison," IEEE Signal Processing Letters, vol. 17, no. 3, pp. 237-240, 2010.

[29] W. W. Tung, J. B. Gao, J. Hu, and L. Yang, "Recovering chaotic signals in heavy noise environments," Physical Review E, vol. 83, no. 4, Article ID 046210, 9 pages, 2011.

[30] R. C. Hwa and T. C. Ferree, "Scaling properties of fluctuations in the human electroencephalogram," Physical Review E, vol. 66, no. 2, Article ID 021901, 8 pages, 2002.

[31] P. A. Robinson, "Interpretation of scaling properties of electroencephalographic fluctuations via spectral analysis and underlying physiology," Physical Review E, vol. 67, no. 3, Article ID 032902, pp. 032902/1-032902/4, 2003.

[32] J. Hu, J. Gao, F. L. Posner, Y. I. Zheng, and W.-W. Tung, "Target detection within sea clutter: a comparative study by fractal scaling analyses," Fractals, vol. 14, no. 3, pp. 187-204, 2006.
[33] J. Hu, W.-W. Tung, and J. Gap, "Detection of low observable targets within sea clutter by structure function based multifractal analysis," IEEE Transactions on Antennas and Propagation, vol. 54, no. 1, pp. 136-143, 2006. 


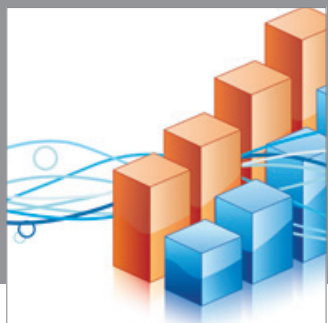

Advances in

Operations Research

mansans

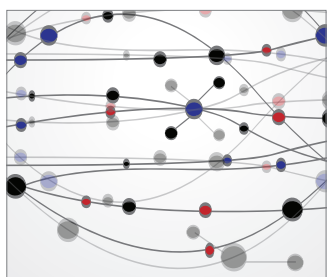

The Scientific World Journal
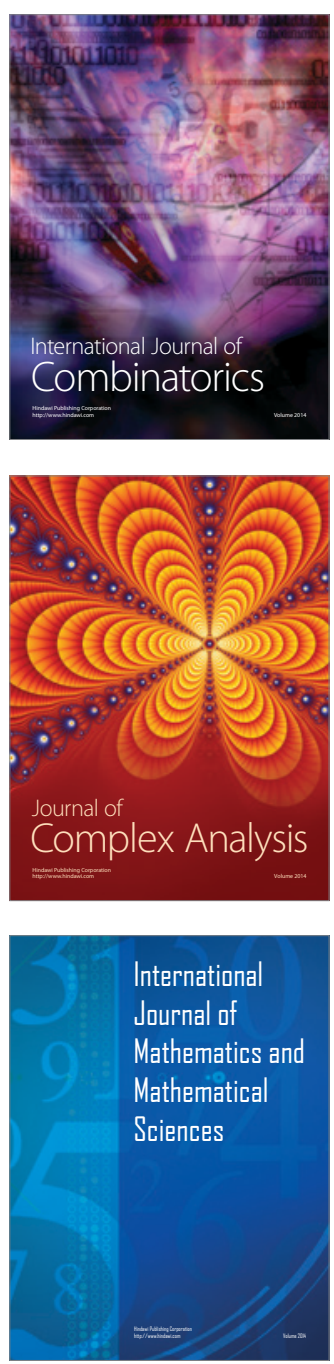
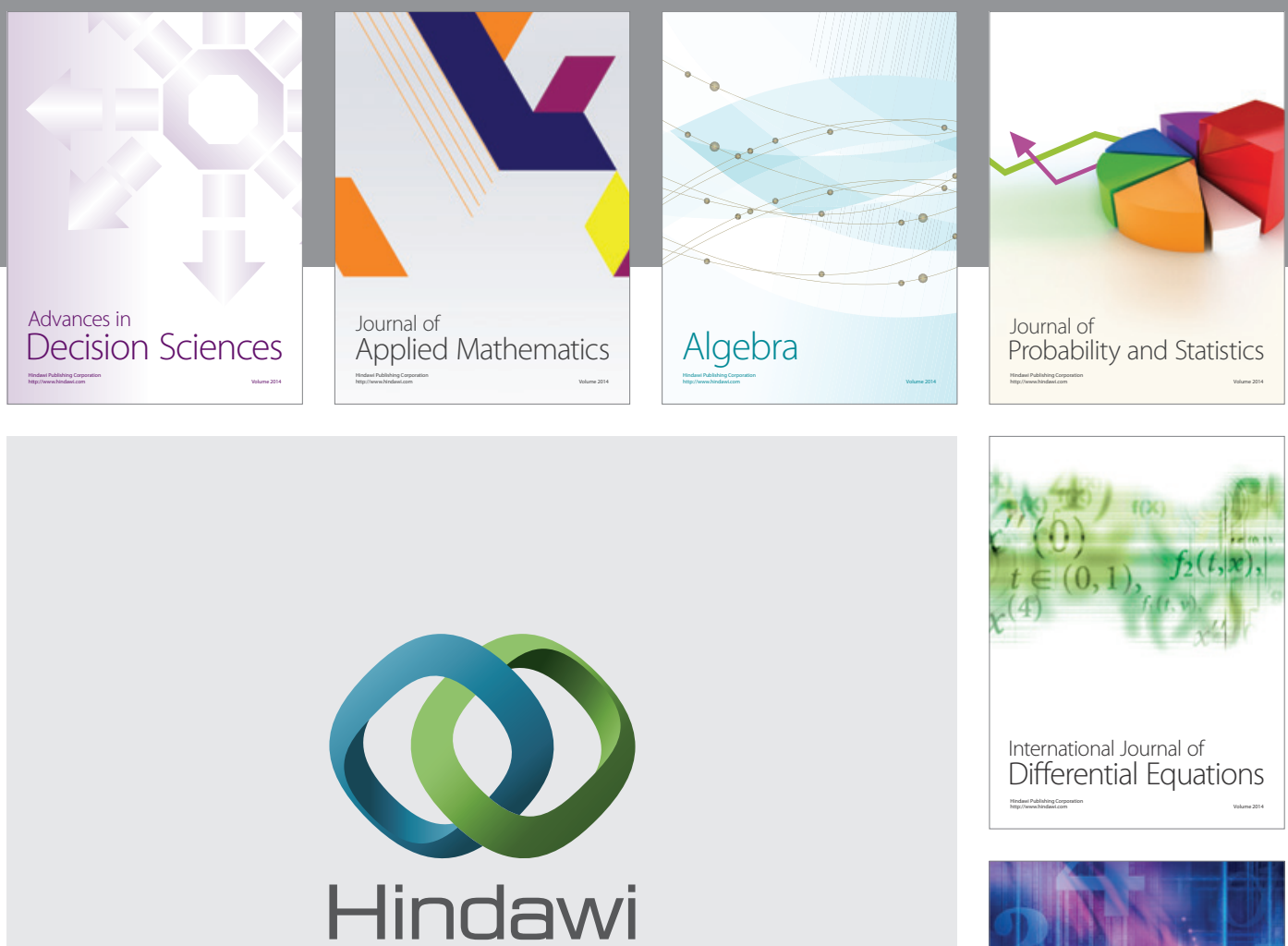

Submit your manuscripts at http://www.hindawi.com
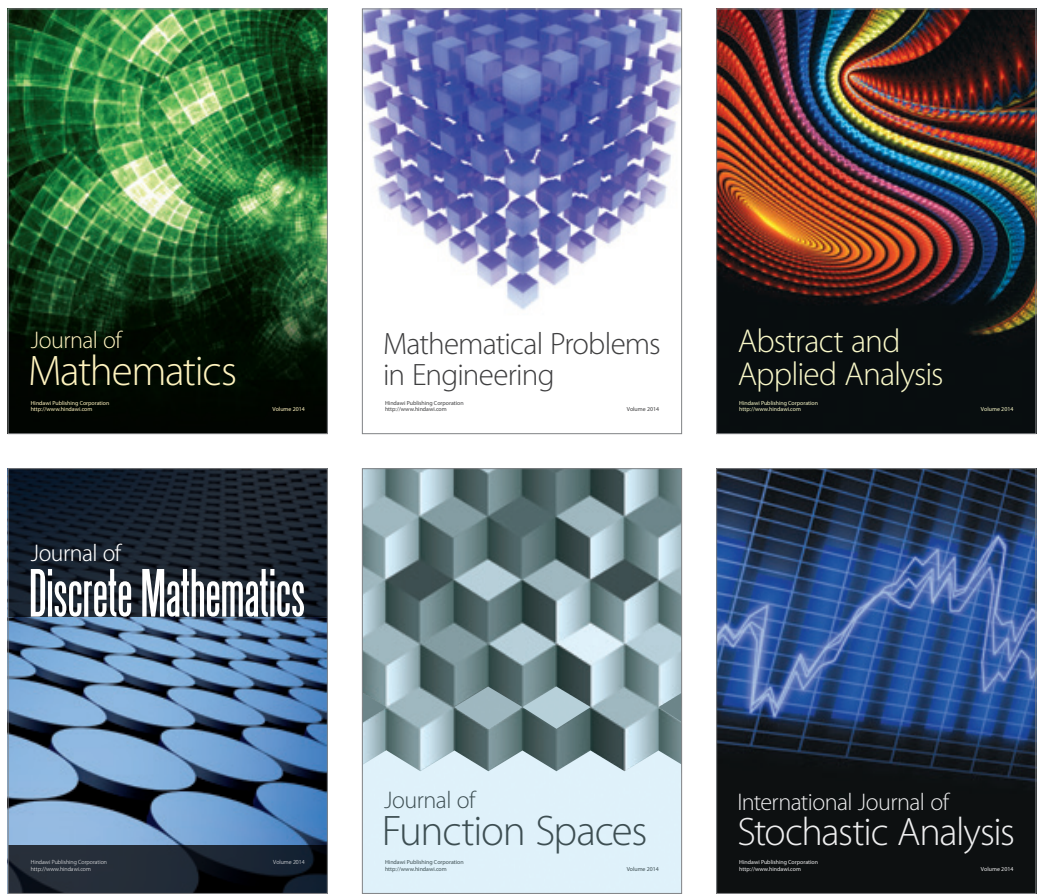

Journal of

Function Spaces

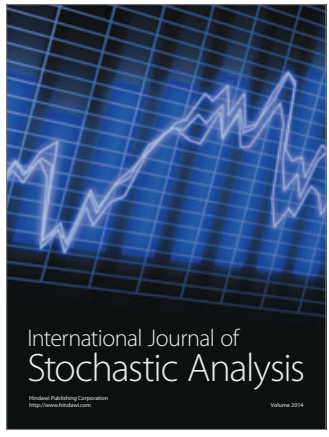

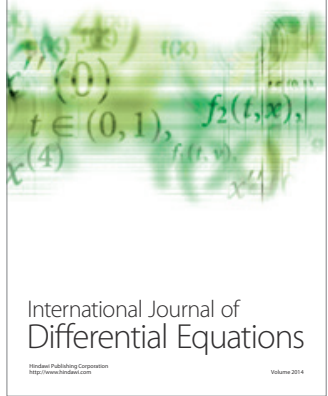
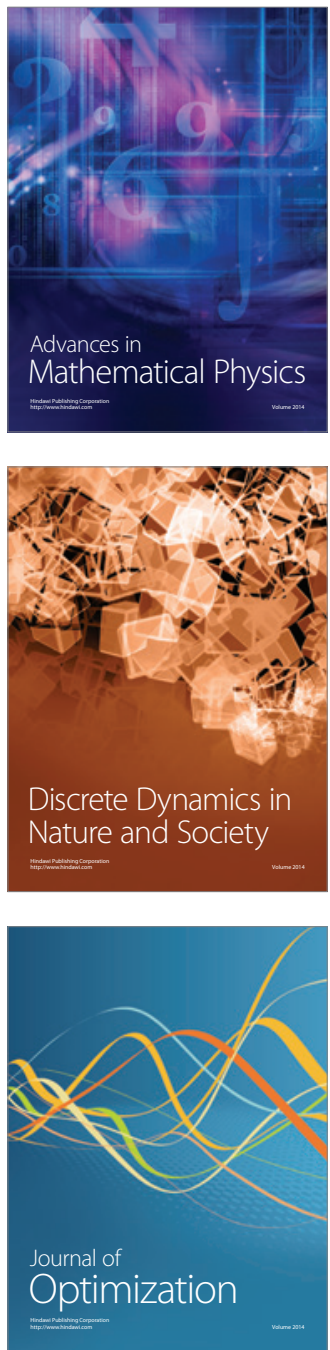